\title{
Microbiological Safety of Using Eye Drops for More Than a Month
}

\author{
Aarthi Rajamanickam¹, Manjula T. Rajendran², Sumangala Basavarajaiah³, Prathibha Shiveshi \\ ${ }^{1}$ Department of Ophthalmology, Mandya Institute of Medical Sciences, Mandya, Karnataka, India. ${ }^{2}$ Department of \\ Ophthalmology, Mandya Institute of Medical Sciences, Mandya, Karnataka, India. ${ }^{3}$ Department of Microbiology, \\ Mandya Institute of Medical Sciences, Mandya, Karnataka, India. ${ }^{4}$ Department of Ophthalmology, Mandya Institute of \\ Medical Sciences, Mandya, Karnataka, India.
}

\section{ABSTRACT}

\section{BACKGROUND}

Contaminated ocular eye drops represent the potential source of preventable ocular infection. The present study was carried out for determining the patterns and magnitude of microbiological contamination of the ocular eye drops in an outpatient clinic in the Ophthalmology Department.

\section{METHODS}

A prospective study was conducted in a tertiary care hospital of Mandya district. A total of 60 eye drop bottles used for more than a month were gathered for nearly 6 months. The samples were assembled from the residual fluid present in the bottle and the tip of the dropper. These collected drops are then inoculated on the plates of different culture media such as blood agar and MacConkey agar, and the resultant growth of microbial organisms were then identified using the standard microbial identification techniques.

\section{RESULTS}

9 out of 60 analyzed bottles showed contamination. The identified bacteria belonged to commensal flora in the eye. The contaminants isolated had coagulase-negative Staphylococci, Acinetobacter and Micrococci. The dropper tips $(\mathrm{n}=6)$ were found to be contaminated more than the residual fluid $(n=1)$. Two $(n=2)$ of the bottles showed contamination in both the tip and residual fluid. The mean contamination percentage for this study is $15 \%$ which is equal to that of other studies.

\section{CONCLUSIONS}

According to our study, the contamination rate was found to be $15 \%$ which specifies the correct handling and proper application of eye drops. The health care professionals or providers should be aware of this and they must educate the patients and the relatives accompanying them about proper usage of the eye drops.

\section{KEY WORDS}

Eye Drops, Contamination, Microbial, Preservatives, Extended Use
Corresponding Author: Dr. Aarthi Rajamanickam, \#50, Victoria Colony, $3^{\text {rd }}$ Street, Medical College Road, Thanjavur-613004, Tamilnadu, India. E-mail: aarthirajamanickam1@gmail.com

DOI: 10.14260/jemds/2019/857

Financial or Other Competing Interests: None.

How to Cite This Article:

Rajamanickam A, Rajendran $M$, Basavarajaiah $S$, et al. Microbiological safety of using eye drops for more than a month. J. Evolution Med. Dent. Sci. 2019;8(52):3964-3967, DOI:
Submission 08-05-2019,

Peer Review 14-12-2019,

Acceptance 20-12-2019,

Published 30-12-2019. 


\section{BACKGROUND}

Extensive utilization of multi-drop ophthalmic eye drops is an arguable problem. In the case of chronic ocular conditions such as allergic diseases, glaucoma, as well as dry eye, the patients are prescribed to use the drops throughout their lives. Solutions or medicines used in Ophthalmology are prone to contamination with microorganisms due to the repetitive usage. ${ }^{1}$ The already conducted studies have depicted the contamination percentage with large variations of $0.07 \%$ and could be extended to $70 \%{ }^{2}$ The fact remains that the amount of contamination increases with increase in usage period of the drops, ${ }^{3}$ whereas not every conducted studies agrees to this fact. ${ }^{4}$ It can also be said that the population of patients can also have an effect, especially in terms of improper use. ${ }^{5}$ Solutions that were free of any preservatives were discovered to be highly prone to contamination as compared to the eye drops with added preservatives. ${ }^{6}$ Usually, the instructions are present on the containers of the eye drops for discarding them in four weeks after opening. In another report, it was concluded that there exists no such difference significantly in the contamination rate between less than 4 weeks and less than 8 weeks of usage.

Another important factor is the location of collection of samples from the bottles of eye drops (cap/tip, Drops, and residual fluid), where the tip has been proved to be the part that was highly contaminated mostly. ${ }^{8}$ The reason behind this could be the lack of preservatives present in this area, as the tip is the area that comes in contact with the lid or the fingers or the cornea and conjunctiva, which possibly leads to contamination even if instilled by healthcare personnel's. In another significant research the authors stated a few cases in Iran where the bacterial keratitis were thought to occur because of eye drops being used on more than one patient. ${ }^{9} \mathrm{On}$ the other hand, some researchers also found that the residual fluid and the drops are contaminated most frequently. The drops when administered using poor techniques are also a factor of risk leading to contamination, specifically for people who are administering their drops by themselves. ${ }^{10}$ Patients who are aged, with poor coordination, poor vision, have fine grasping difficulty, and lower coordination abilities might touch the skin or eyes with the dropper resulting in contamination ${ }^{11}$ Preservatives used in the eye drops must meet following necessity: (1) should be synergetic to other ingredients; (2) should be efficacious throughout the usage duration of the eye drops; and (3) should be harmless for eyes. Basically, there exist two mechanisms that make the preservatives work, they either act as per the oxidation process or works as detergents. Detergents act by dissolving or disrupting lipids. Example of which includes benzalkonium chloride, polyquaternium-1(PQ-1), preservatives in alcoholic as well as phenol. The oxidation preservatives result in oxidative reactions for disrupting the metabolism of the cells. Examples of which include thiomersal, sodium perborate, sorbic acid, chlorhexidine. As per a report, the bottles made of plastic were contaminated quite commonly near the cap of the bottle. It can be linked to the lower quantities of preservatives on that area. ${ }^{12}$ Preservatives as such will interfere with the metabolism of the existing microorganism and inhibits their growth, they also have similar effects on conjunctival and cornea epithelial cells causing apoptosis of those cells, decreasing quantity of the cells of the goblet cells, and increasing inflammatory marker expressions on the conjunctival epithelial cells, resulting in problems such as dry eye and other ocular surface diseases. Hence, the ocular eye drops might increase the risk of infection, specifically in case of a compromised ocular epithelial barrier. It is highly important to reduce the eye drop contamination as well as low infection transmission in the case of clinical ophthalmology.

The present study aimed at determining the patterns and magnitude of microbiological contamination of ocular eye drops at an outpatient clinic in the Ophthalmology Department.

\section{METHODS}

A prospective study was conducted in a tertiary care hospital of Mandya district. Nearly 60 bottles of eye drops used for more than a month were collected for nearly 6 months. The size of the sample used was dependent on the study convenience. The set of samples were gathered from the residual fluid and tip of the dropper of the bottle. Different culture plates were used for inoculation of the drops such as blood agar and MacConkey agar, where the microbial growth outcome is identified as per the standard techniques of microbial identification. The eye drops bottles with the minimum usage of 1 month were collected from the patient. No restrictions were made in terms of the disease or the drug. The usage period was further noted. Eye drop containers having a sufficient amount of residual solutions were sorted out. The external appearance of the bottle was visually observed as "unclean" or "clean". The bottle was categorized as unclean in case any part of the body, tip, or the neck of the bottle was dirty. The bottles were then de-labelled and coded after collection (specific lab numbers were given to the bottles). Before sampling the eye drop bottles were stored at $48^{\circ} \mathrm{C}$ for a maximum of 24 hours and then sent for microbiological assessment in the Department of Microbiology. A sterile swab is rolled over the tip and inoculated on MacConkey agar and blood agar media, incubated overnight. Loopful of residual fluid is inoculated on blood agar and another loopful on MacConkey agar, incubated for 48 hours on $37^{\circ} \mathrm{C}$ and were evaluated after 24 and 48 hours. The blood-agar, chocolate-blood agar plates are incubated in a microaerophilic environment. Growth if observed is processed as per the guidelines ${ }^{13}$. Ethical Committee clearance was obtained before starting the study

\section{Statistical Analysis}

The Statistical Package for Social Science \{SPSS Version 20 will be used for Data Analysis Mean, median, and SD are used to describe quantitative data. Qualitative data are summarized using frequency and percentage.

\section{RESULTS}

Overall 60 eye-drop-bottles were collected in the outpatient department and examined for the microbiological contamination, out of which $9(15 \%)$ bottles were found to be 
contaminated at the bottle tip alone or with additional contamination of the solutionthe eye drop bottles were grouped according to their contents. 1. Lubricants 2 . Antibiotics 3. Antibiotic + steroid 4. Local anaesthetic 5 . Antibiotic +NSAIDS 6. Anti-glaucoma 7. NSAIDS 8. Steroids. Lubricants showed maximum (33\%) contamination rate followed by Antibiotic +NSAID (25\%), Antibiotic + steroid (12.5\%), plain Antibiotics (11\%) and rest showed no contamination. The dropper tip was found to be contaminated more, six $(n=6)$ of the bottles had contaminants than the residual fluid which was contaminated in one $(n=1)$ bottle, where two $(n=2)$ bottles showed contamination in both the tip and residual solution. All the tested eye drops in our study contained preservatives.

\begin{tabular}{|c|c|c|}
\hline No. & Type of Preservative & No. of Medications \\
\hline 01 & Benzalkonium chloride $0.05 \%$ & 26 \\
\hline 02 & Benzalkonium chloride $0.02 \%$ & 12 \\
\hline 03 & Benzalkonium chloride $0.01 \%$ & 9 \\
\hline 04 & Thiomersal $0.005 \%$ & 7 \\
\hline 05 & Stabilized oxychloro complex & 6 \\
\hline \multicolumn{2}{|c|}{ Table 1. Preservatives Used and Their Frequency of Eye Drops } \\
\hline \multicolumn{2}{|c|}{ Contamination } \\
\hline
\end{tabular}

\begin{tabular}{|c|c|c|}
\hline Preservative & No. of Medications & \%age of Contamination \\
\hline Benzalkonium chloride $0.05 \%$ & 26 & $4(15.38 \%)$ \\
\hline Benzalkonium chloride $0.02 \%$ & 12 & $2(16.66 \%)$ \\
\hline Benzalkonium chloride $0.01 \%$ & 9 & $1(11.11 \%)$ \\
\hline Thiomersal $0.005 \%$ & 7 & $1(14.28 \%)$ \\
\hline Stabilized oxychloro complex & 6 & $0(0 \%)$ \\
\hline \multicolumn{3}{|c|}{ Table 2} \\
\hline
\end{tabular}

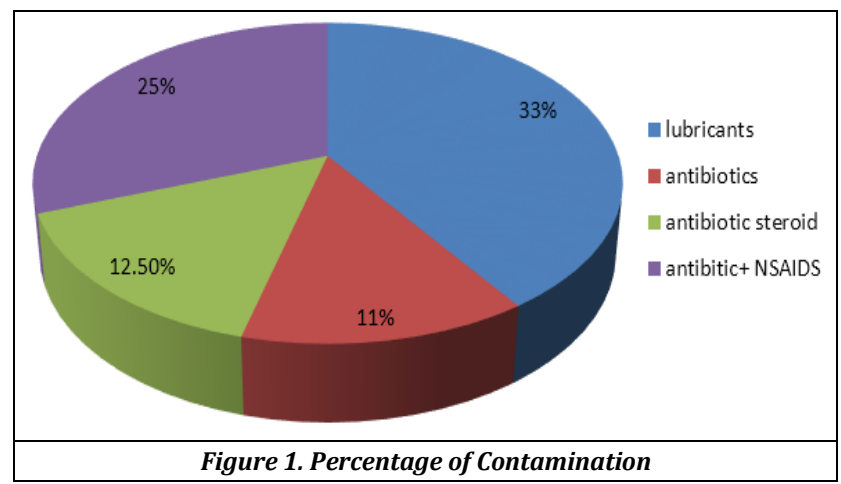

\begin{tabular}{|c|c|c|c|c|c|c|}
\hline 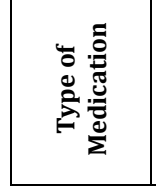 & 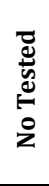 & 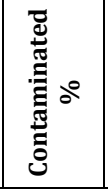 & 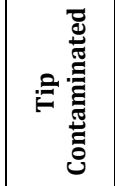 & 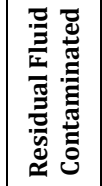 & 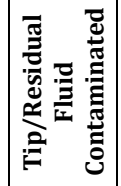 & 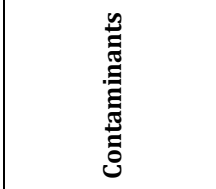 \\
\hline Lubricants & 15 & $5(33 \%)$ & $4(26.6 \%)$ & $1(6.6 \%)$ & - & \begin{tabular}{|c}
$\begin{array}{c}\text { Coagulase negative } \\
\text { staphylococci } \\
\text { Acinetobacter species }\end{array}$ \\
\end{tabular} \\
\hline Antibiotics & 18 & $2(11 \%)$ & - & - & $2(11 \%)$ & \begin{tabular}{|c|} 
Coagulase negative \\
staphylococci \\
Micrococci
\end{tabular} \\
\hline $\begin{array}{c}\text { Antibiotic+ } \\
\text { steroid }\end{array}$ & 8 & $1(12.5 \%)$ & $1(12.5 \%)$ & - & - & $\begin{array}{c}\text { Acinetobacter } \\
\text { species }\end{array}$ \\
\hline $\begin{array}{c}\text { Local } \\
\text { anaesthetic }\end{array}$ & 4 & 0 & 0 & - & - & spowes \\
\hline $\begin{array}{c}\text { Antibiotic+ } \\
\text { NSAIDS } \\
\end{array}$ & 4 & $1(25 \%)$ & $1(25 \%)$ & - & - & $\begin{array}{c}\text { Acinetobacter } \\
\text { species }\end{array}$ \\
\hline \begin{tabular}{|l|} 
Antiglaucoma \\
\end{tabular} & 4 & 0 & 0 & - & - & - \\
\hline NSAID & 4 & 0 & 0 & - & - & - \\
\hline Steroids & 3 & 0 & 0 & - & - & - \\
\hline Total & 60 & $9(15 \%)$ & $6(10 \%)$ & $1(2 \%)$ & $2(3 \%)$ & - \\
\hline
\end{tabular}

The organisms identified were part of the normal conjunctival and skin flora. Gram positive bacteria like coagulase negative Staphylococci, Micrococci and gramnegative Acinetobacter species. Coagulase negative Staphylococcus were found in 6/9 (6 out of 9) of the bottles, Acinetobacter were found in two of the bottles and Micrococci was found in one of the bottles being tested. Two of the medications showed more than one contaminant. Four of the 60 bottles were found to be past the expiry date. None of them showed any contamination.

Data on the appearance of the bottles and the number of days the bottles had been in use were available for 53 of the 60 bottles tested. The 12 bottles were classified as unclean and 48 bottles classified as clean. Visual inspection only identified two (22\%) of the 9 contaminated solutions as having dirty bottles. Contaminated solutions were present in one $(8 \%)$ unclean bottle and seven (14\%) clean bottles.

Compared with bottles classified as clean, unclean bottles were 1.75 times more likely to be contaminated with bacteria.

\section{DISCUSSION}

In our study, we found contamination of about $15 \%$ ( 9 out of 60 bottles). We detected three different micro-organisms (Gram-positive Coagulase-negative staphylococci and micrococci, Gram-negative Acinetobacter). All the organisms present were the part of normal commensal flora of the skin or the conjunctiva. The eyelid and conjunctiva of the resident flora are comprised of CONS (Coagulase Negative Staphylococci), Enterobacter spp, Micrococcus spp, Bacillus spp, Propionibacterium spp, Corynebacterium spp, as well as Staphylococcus aureus. ${ }^{14}$ We did not find any pathogenic organisms in our study.

The contamination cycle exists between dropper tips and the lids which was previously suggested by Schein at al.15 Contaminated eye drop dispenser or the eye drops having a similar kind of micro-organisms, especially of Gram-negative type was described by the same group. It simply represents a serious potential risk of ocular infection, mainly when the corneal epithelium is compromised due to excessive wearing of contact lenses, excess use of topical steroids, or ocular trauma Both dropper tip $(n=6)$ and residual fluid $(n=1)$ showed contamination in our study with contamination of both residual fluid and tip in two $(n=2)$ bottles. The received outcomes correspond to all of the results mentioned in the previously conducted studies. ${ }^{16}$ The main reason for consideration is the antimicrobial susceptibility of preservatives of the solution. However, such antimicrobial effects might not optimally act on its tips as the time of contact is quite limited.

In addition, the tip provides a large surface area for contamination either from hands or ocular structures. In a few instances, dry crusts are also seen on the bottle tips. The disposal of these residues using a sterile swab can help in reducing the further rate of contamination. Although, the solution when contaminated is regarded to be clinically relevant as they come in direct contact with patient's eye.

The contamination of the solution is determined by various factors. Usage pattern, type of drug, time past after opening, absence or presence of preservative, and sample source are the reasons which can have an impact on the culture results. In studies where the collection of samples were 
done from the solution only, the results show a low incidence of contamination or either it was not detected. ${ }^{17}$ Nentwich et al did not discover any difference significantly in contamination rate between the residual solution and the tip of the dropper.

\section{Possible Causes of Microbial Contaminations}

- Failure of prescriber to inform the patient about proper use of eye drop preparations. All the prescribers did explain the precautions to patients, but the older age of patients, lower educational status and the noisy ambience of government OPD made the work difficult.

- Failure of patients to follow the precautions of eye drop use- when inquired authors found that many patients did not follow the precautions, but on analysis authors could not found any direct correlation between findings of contamination and not taking the aseptic precautions.

- Failure of preservative added to the eyedrop- the guidelines of using an eye drop up to four weeks after opening, is based on proper functioning of the preservative used.

Authors found that contamination was occurring irrespective of use of preservative, and the principal content of the preparation. Only eye drops containing antimicrobial agents, without any additional glucocorticoids were having the least incidence $(6.66 \%)$ of contaminations. Saisyo et al in his study, showed that eye drops without preservatives had zero risk of oculo-toxic effects and lack of microbial contamination. ${ }^{18}$

\section{CONCLUSIONS}

According to our study, the contamination rate was found to be $15 \%$ which specifies the correct handling and proper application of eyedrops. The health care professionals or providers should be aware of this and they must educate the patients and the relatives accompanying the patients about proper handling and administration of the eye drops.

\section{REFERENCES}

[1] Jokl DH, Wormser GP, Nichols NS, et al. Bacterial contamination of ophthalmic solutions used in an extended care facility. $\mathrm{Br} \quad \mathrm{J}$ Ophthalmol 2007;91(10):1308-10.

[2] Nentwich MM, Kollmann KH, Meshack J, et al. Microbial contamination of multi-use ophthalmic solutions in Kenya. Br J Ophthalmol 2007;91(10):1265-8.

[3] Fazeli MR, Nejad HB, Mehrgan H, et al. Microbial contamination of preserved ophthalmic drops in outpatient departments: possibility of an extended period of use. DARU Journal of Pharmaceutical Sciences 2004;12(4):151-5.

[4] Livingstone DJ, Hanlon GW, Dyke S. Evaluation of an extended period of use for preserved eye drops in hospital practice. Br J Ophthalmol 1998;82(5):473-5.

[5] Porges Y, Rothkoff L, Glick J, et al. Sterility of glaucoma medications among chronic users in the community. J Ocul Pharmacol Ther 2004;20(2):123-8.

[6] Rahman MQ, Tejwani D, Wilson JA, et al. Microbial contamination of preservative free eye drops in multiple application containers. Br J Ophthalmol 2006;90(2):13941.

[7] Geyer O, Bottone EJ, Podos SM, et al. Microbial contamination of medications used to treat glaucoma. $\mathrm{Br}$ J Ophthalmol 1995;79(4):376-9.

[8] Hovding G, Sjursen H. Bacterial contamination of drops and dropper tips of in-use multidose eye drop bottles. Acta Ophthalmol (Copenh) 1982;60(2):213-22.

[9] Jalali R, Zinolabedini F, Moradi M, et al. Bacterial contamination rate of eyedrops: comparison of a hospital and a private outpatient center in Kermanshah, Iran. Insight 2004;29(3):12-4.

[10] Ginés JC, Samudio M, Fariña N, et al. Microbial contamination of eye drops used by patients in an outpatient clinic of ophthalmology. Memorias del Instituto de Investigaciones en Ciencias de la Salud 2012;10(2):38-46.

[11] Teuchner B, Wagner J, Bechrakis NE, et al. Microbial contamination of glaucoma eyedrops used by patients compared with ocular medications used in the hospital. Medicine 2015;94(8):e583.

[12] Clark PJ, Ong B, Stanley CB. Contamination of diagnostic ophthalmic Solutions in primary eye care settings. Mil Med 1997;162(7):501-6.

[13] Collee JG, Fraser AG, Marmiom BP, et al. Practical Medical Microbiology. 14th edn. India: Elsevier 2008: p. 131-49.

[14] Leong JK, Shah R, McCluskey PJ, et al. Bacterial contamination of the anterior chamber during phacoemulsification cataract surgery. J Cataract Refract Surg 2002;28(5):826-33.

[15] Schein OD, Hibberd PL, Starck T, et al. Microbial contamination of in-use ocular medications. Arch Ophthalmol 1992;110(1):82-5.

[16] Coad CT, Osato MS, Wihelmus KR. Bacterial contamination of eye drop dispensers. Am J ophthalmol 1984,98(5):548-51.

[17] Prabhasawat P, Chotikavanich S, Leelaporn A. Sterility of non-preservative free eye drops. J Med Assoc Thai 2005;88(Suppl 9):S6-10.

[18] Saisyo A, Oie S, Kimura K, et al. Microbial contamination of in-use ophthalmic preparations and its prevention. Bull Yamaguchi Med Sch 2016;63(1-2):17-24. 